\title{
Untersuchungen über die Windverhältnisse in der Nordwestschweiz
}

\section{Problemstellung}

Zur Beurteilung der Möglichkeit einer klimatischen Veränderung durch eine größere Zahl von Kernkraftwerken wurden im Projekt CLIMOD (1981) zahlreiche Messungen von Wind, Temperatur und Feuchtigkeit durchgeführt. Einige Ergebnisse sollen hier dargestellt werden mit Hinweis auf neue Fragen, die sich daraus ergeben.

Je intensiver die menschliche Tätigkeit ist, desto mehr entstehen kontinuierlich oder bei Unfällen Abgase, die entfernt werden müssen. Wie rasch diese Luftverunreinigungen weggetragen oder verdünnt werden oder auch ausfallen, hängt von den Windverhältnissen und der vertikalen Temperaturverteilung ab. Da Temperatur und Wind von der Wetterlage abhängig sind, muß man versuchen, die Unzahl der möglichen Wettersituationen in eine überschaubare Anzahl von typischen regionalen Wetterlagen "TRW» zu gruppieren. Leider hat sich die Hoffnung nicht bestätigt, daß man mit 6 Wetterlagen und einer Unterscheidung zwischen Tag und Nacht, Sommer und Winter, also insgesamt 24 Gruppen, eine eindeutige Beschreibung der Strömungsverhältnisse erhalten könne. Wohl kann in vielen Fällen über längere Strecken mit über $50 \%$ Wahrscheinlichkeit eine Windrichtung angegeben werden BROMBACHER (1979); aber es gibt sowohl Stationen mit schwachen Winden aus unbestimmter Richtung als auch Fälle, wo der Wind aus einer ganz anderen Richtung kommt (solche Fälle werden als Alternativ-Situation bezeichnet). Somit kann die vorliegende Arbeit noch keine abschließenden Resultate vorlegen; vielmehr ergeben sich Hinweise, in welcher Richtung die Forschung weitergetrieben werden muß.

\section{Darstellungsmethode}

Nur vier der 48 Beispiele können hier abgebildet werden. Eine Reliefdarstellung, von M. wINIGER entworfen, zeigt von Westen her gesehen im Vordergrund das Rheinknie in Basel, rechts Birsig und Birs; links den Rhein bis zum Isteinerklotz. Im Mittelfeld sehen wir von links nach rechts das Wiesental, den Dinkelberg mit St. Chrischona, den Hochrhein, das Gempenplateau und östlich davon das Ergolztal. Im obern Drittel ragt links der Hotzenwald bis auf $1000 \mathrm{~m}$ im NW empor und fällt abrupt zum Hochrhein bei Stein-
Säckingen; nach rechts erkennt man das Fricktal, dem der $750 \mathrm{~m}$ hohe Tiersteinberg vorgelagert ist. Zuoberst erkennt man links den Hochrhein bei Leibstadt, von rechts nach links ist teils der Durchbruch der Aare durch den Jura erkenntlich.

Auf dieses Relief werden nun die Windströmungen in drei Höhenniveaus aufgetragen. Die voll ausgezogene Linie zeigt die Strömung im Talgrund; die dünnere Doppellinie stellt die Strömung in mittlerer Höhe (500 bis $700 \mathrm{~m} \mathrm{NN}$ ) dar, und schließlich ist die Windrichtung auf dem Feldberg ( $1500 \mathrm{~m} \mathrm{NN})$ durch gerade parallele Doppellinien als Hinweis auf die Strömung in der Freien Atmosphäre dargestellt. Die Annahme, $\mathrm{da} B$ die Windrichtung vom Feldberg für das ganze dargestellte Gebiet gelte, ist nicht immer erfüllt. Soweit in mehr als der Hälfte der Meldungen der Wind aus einer bestimmten Richtung weht, sind die Stromlinien zusammenhängend dargestellt. Bei 30 bis $40 \%$ Wahrscheinlichkeit sind sie gestrichelt und bei 20 bis 30\% noch punktiert dargestellt. Da die vertikale Durchmischung umso stärker ist, je rascher die Temperatur mit der Höhe abnimmt, ist links oben noch ein Temperaturprofil zwischen 200 und $1500 \mathrm{~m}$ Höhe beigefügt. Dort zeigt die Diagonale die «neutrale» Luftschichtung (Trockenadiabate), welche Vertikalbewegungen nicht behindert; in diesem Falle sind die Strömungsverhältnisse vor allem durch Reibungskräfte bestimmt. Ist dagegen (vor allem bei klarer Nacht) die Luftschichtung stabil oder herrscht gar Inversion (Temperatur nimmt mit der Höhe zu), dann sind Luftströmungen weitgehend durch die Topographie geprägt.

\section{Winter, Nordlage (Fig. 1)}

Bei der gemäß der TRW-Klassifikation mit $\mathrm{E}$ bezeichneten Nordlage beobachtet man im Winter nachts im Hochrheintal von Basel bis Leibstadt eine Westströmung sowohl im Tal als auch über Chrischona $(500 \mathrm{~m})$ und Gempen $(700 \mathrm{~m})$ und auch über dem Feldberg $(1500 \mathrm{~m})$. Überraschenderweise weht auch in den Seitentälern (Birs, Ergolz, Frick) der Wind talaufwärts und geht über die Jurapässe Wasserfallen $(1000 \mathrm{~m})$

\section{PD Dr. Walter Schüepp}

Abteilung für Meteorologie, Postfach

$\mathrm{CH}-4010$ Basel 10 
und Salhöchi ( $800 \mathrm{~m})$ ins Mittelland hinüber, während gleichzeitig im Elsaß ein Zweig als Südwind talabwärts strömt. Man kann sich gut vorstellen, daß sich die über die Burgundische Pforte einströmende Luft an Jura und Schwarzwald staut und deshalb ein Teil nach Süden über die Jurapässe und ein Teil nach Norden durch die Oberrheinische Tiefebene ausweicht. Offen bleibt die Frage, warum bei Nordlage der Wind über Basel offensichtlich nicht von Norden, sondern von Westen her kommt.

\section{Winter, Südlage (Fig. 2)}

Bei der gemäß TRW-Klassifikation mit $\mathrm{F}$ bezeichneten Südlage erwartet man das Gegenteil der in Fig. 1 dargestellten Nordlage. Tatsächlich weht im Winter nachts im Hochrheintal die Strömung vom Talgrund bis auf die Höhe des Feldbergs aus Osten und fließt dann teils in die Oberrheinische Tiefebene hinunter und teils durch die Burgundische Pforte westwärts. In den Seitentälern (Frick, Ergolz, Birs) weht der Wind hangabwärts und talauswärts, wie dies für nächtlich abgekühlte Luft schon allein wegen der Schwerkraft sein sollte. Auch daß bei Südlage der Wind über die Jurapässe nach Norden strömt, entspricht den Erwartungen. Während diese Winde im allgemeinen ziemlich schwach sind, bildet sich in der Gegend von Säckingen-Möhlin-Karsau eine ungewöhnlich starke Strömung aus, die von der Forschergruppe CLIMOD mit «Möhlin-Jet» benannt wurde. Diese Strömung weht schief zum Tal. Der Möhlin-Jet hat offensichtlich seine Quelle im Nebelmeer, das über dem schweizerischen Mittelland liegt. SCHÜEPP (1980). Das Hochrheintal ist die tiefste Rinne, durch welche die im Mittelland entstandene Kaltluft ausfließen kann. Wegen der Talenge in der Gegend von Säckingen zwischen dem Hotzenwald im Norden und dem Tiersteinberg im Süden kommt es zu einer Düsenwirkung, worauf der 300 bis $500 \mathrm{~m}$ mächtige Stromschlauch unbeachtet der Hindernisse geradeaus schießt, bis er langsam seitlich ausfächert - ein kleiner Teil strömt Richtung Kaiseraugst, die Hauptmasse streicht über das Plateau des Dinkelbergs, überquert das parallel zum Hochrhein liegende Wiesental und erreicht die Oberrheinische Tiefebene etwa bei Kembs. Während im Rheintal bei Basel die Luft nachts sehr stabil ist und in diesem Zustand genau den Talrinnen folgen muß, zeigt der Möhlin-Jet in seinem Kern eine beinahe adiabatische Luftschichtung, d.h. die Temperatur nimmt etwa $1 \mathrm{Grad}$ pro 100 m Höhe ab; darum kann er ohne Energieverlust über den Dinkelberg aufsteigen. Eine adiabatische Temperaturschichtung kann nachts nur unter einer Hochnebeldecke bestehen bleiben; die Tatsache, daß die über den Fricktalerjura einströmende Hochnebeldecke sich nicht auflöst, ist also wesentlich für die volle Entwicklung des Möhlin-Jets. Umgekehrt ist die Gegend rund um Basel zwischen Kembs-Lörrach-Rheinfelden-Rothenfluh-Aesch bei dieser Wet- terlage durch Nebelarmut gekennzeichnet. Dabei ist die Temperatur nahe der Bodenoberfläche nahezu die gleiche wie am Grunde des Möhlin-Jets, nach oben aber bleibt sie im nebelfreien Gebiet konstant oder nimmt gar mit der Höhe zu; somit ist es auf dem nebelfreien Gempen 4 bis 6 Grad wärmer als auf der gleich hohen Salhöchi im Nebel aus dem Mittelland. Von $1000 \mathrm{~m}$ an aufwärts herrscht meist gute Fernsicht bei föhniger Bewölkung und nur schwachen Winden. Bei Muttenz erreicht die Strömung im Hochrheintal einen zweiten Höhepunkt, wohl als Sammlung der Strömungen von Rheinfelden her und aus dem Ergolztal.

Interessanterweise bildet sich in etwa einem Drittel der Fälle bei Wetterlage $F$ in mittlerer Höhe (Chrischona, Gempen), aber auch im obern Teil der Täler (Leibstadt, Sissach), eine Gegenströmung, d.h. ein Westwind aus, während am Talgrund der Talabwind und in Feldberghöhe der Ostwind bestehen bleiben. Es ist dies eine "Alternativsituation", deren Entstehungsursache noch nicht geklärt ist.

\section{Sommer, Hochdrucklage, nachts (Fig. 3)}

Neben diesen entgegengesetzten Winterlagen wollen wir einen Blick in die Verhältnisse im Sommer tun. Da im Sommer die synoptischen Winde schwächer sind, andererseits besonders während des langen Tages die Strahlung die Temperaturverhältnisse viel stärker verändert, soll die Hochdrucklage in den Vordergrund gestellt werden.

Bei der mit TRW A bezeichneten Hochdrucklage sind die Winde meist schwach, wobei nachts auf dem Feldberg häufig eine westliche Komponente beobachtet wird, tagsüber dreht dann der Wind auf Ost und zeigt damit an, daß im Sommer der Tagesgang der Strahlung die Strömungsverhältnisse bis über die Feldberghöhe hinaus beeinflußt. Nur in der untersten Luftschicht bis etwa $500 \mathrm{~m}$ herrscht eindeutig Inversion mit der tiefsten Temperatur am Talgrund. Darüber sind die Luftmassen recht stabil geschichtet und müssen damit weitgehend der Topographie folgen als Hangab- und Bergwinde. Mit großer Regelmäßigkeit weht nachts der Wind talauswärts und in die Oberrheinische Tiefebene hinunter. Zum Teil verstärkt sich auch im Sommer noch die Strömung in der Gegend von Möhlin mit einer Drehung Richtung Dinkelberg, sie ist jetzt aber wesentlich schwächer und unbeständiger als im Winter. Die Kaltluftmassen im Mittelland können sich nicht so hoch aufstauen, da sie tagsüber jeweils wieder aufgewärmt werden. Entsprechend reicht die rheinabwärts gerichtete Strömung im Sommer nur wenig über Chrischona hinaus, während das Gempenplateau bereits häufig in der Gegenströmung aus West bis Nordwest liegt, die über die Wasserfalle ins Mittelland hinüberzieht. Die niedrigeren Pässe Staffelegg $(600 \mathrm{~m})$ und Salhöchi $(800 \mathrm{~m})$ werden nachts dagegen noch häufig vom Mittelland her nach Norden überflossen. 

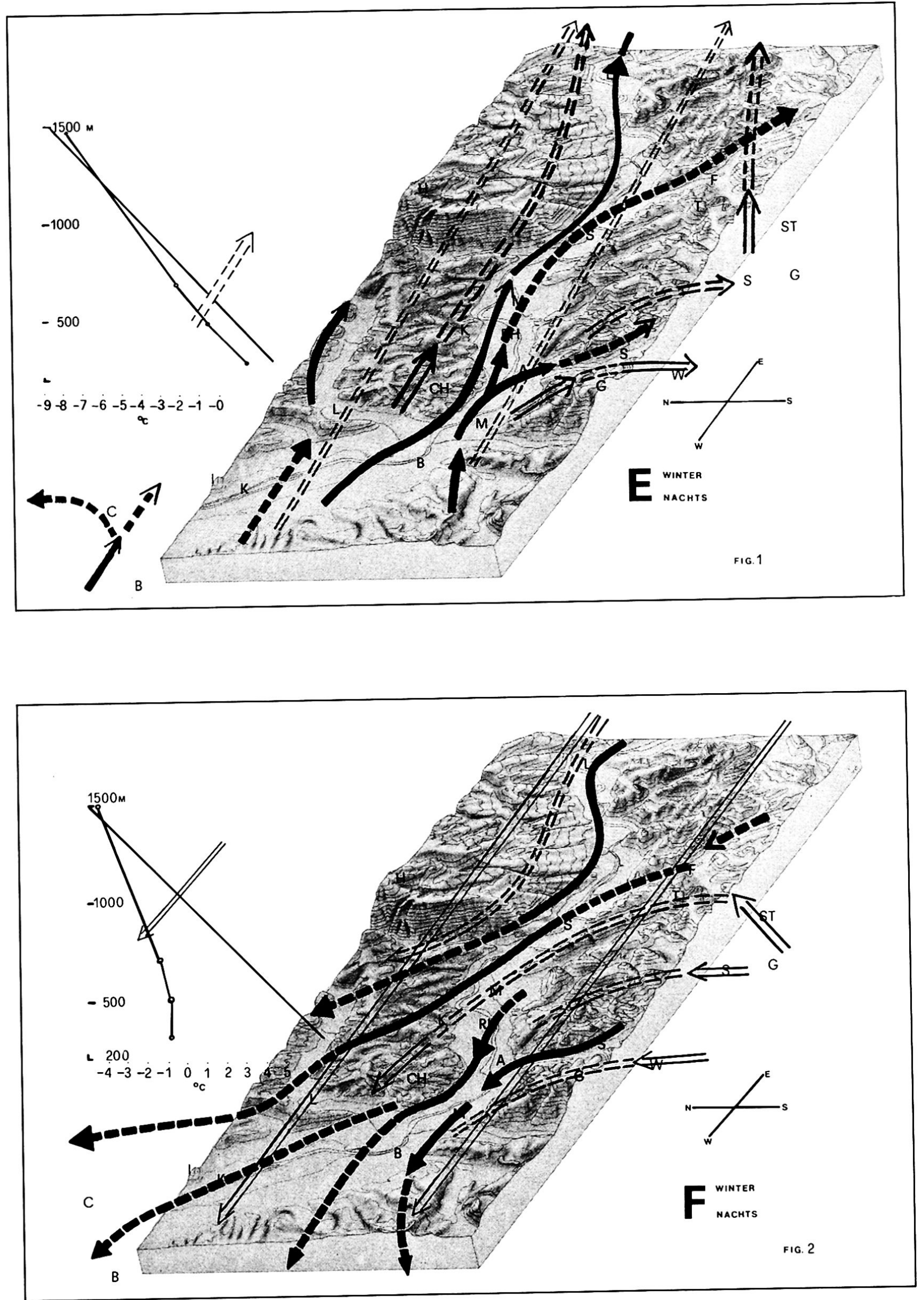

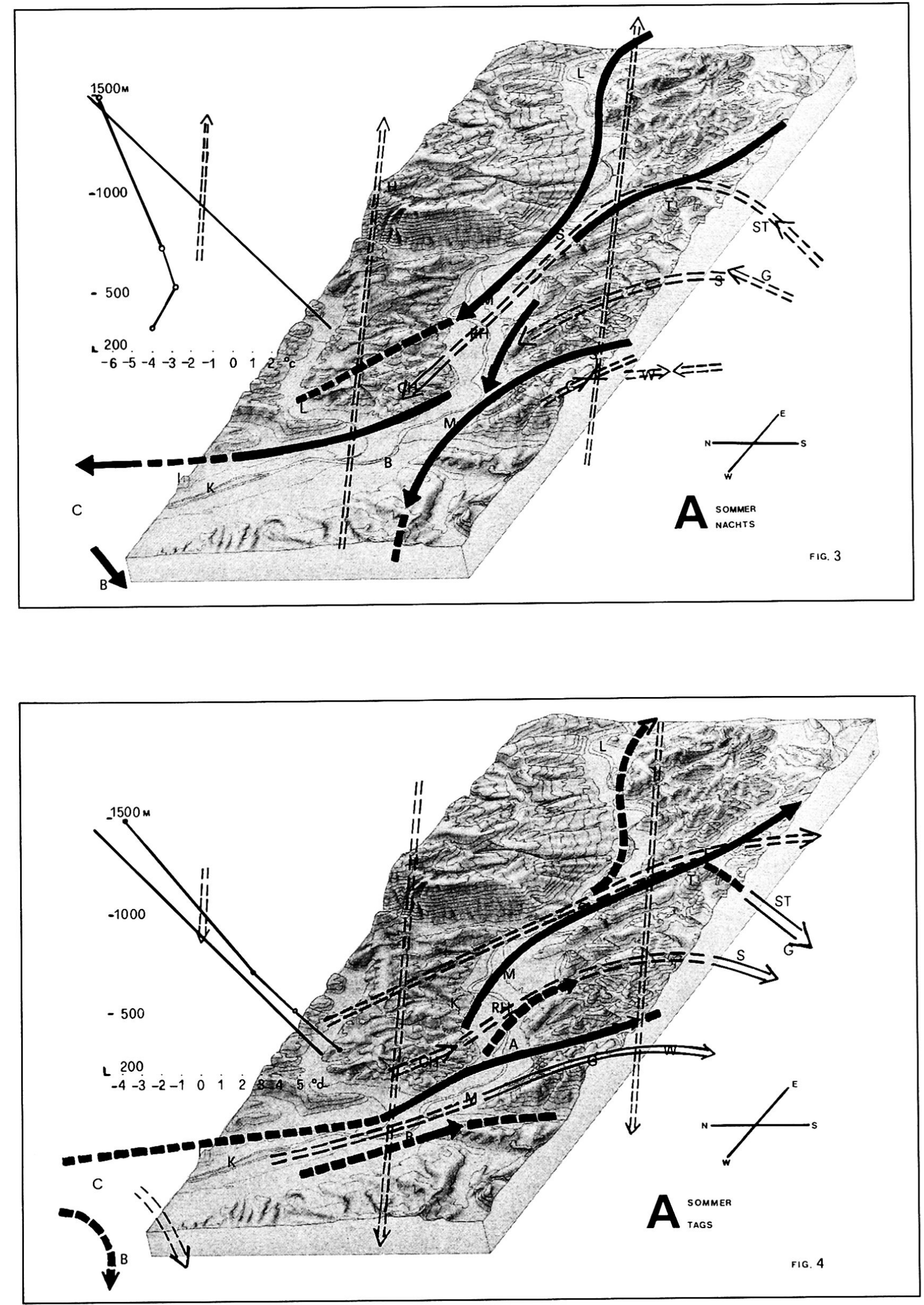
Nicht selten setzt sich auch hier eine "Alternativlage" durch, wobei die Strömung von Colmar bis Leibstadt rheinaufwärts verläuft und auch über die Jurapässe ins Mittelland hinüber gelangt.

\section{Sommer, Hochdrucklage, tags (Fig. 4)}

Anders liegen die Verhältnisse bei der Hochdrucklage TRW A im Sommer tagsüber. Durch die intensive Strahlung hat sich die ganze Luftschicht bis über die Höhe des Feldbergs hinaus erwärmt, so daß praktisch eine adiabatische Luftschichtung herrscht, welche den Vertikalbewegungen keinen Widerstand entgegensetzt. Auf dem Feldberg dominiert eine Ostströmung, während die übrigen Stationen generell eine Nordwestströmung erkennen lassen. Diese führt die Luft einerseits von der Oberrheinischen Tiefebene in Richtung auf den Jura und andererseits ins Hochrheintal hinein. Obwohl die Hauptjuratäler nach Norden abfallen und deshalb nicht so intensiv bestrahlt sind wie der steile Jurasüdhang, hat die Talaufströmung im Ergolz- und Fricktal offenbar mehr Energie, mehr Masse, so daß nachmittags alle drei Pässe von Nord nach Süd überflossen werden. Dabei kommt es auf der Südseite, abgesehen von einer dünnen Hangaufwindschicht, zu einer leicht absinkenden Luftbewegung. Dies zeigt sich deutlich in der Abnahme der Bewölkung über dem Aaretal und an der Richtung der dann kurzen Dampffahne am Kühlturm bei Gösgen. Die von der Rheinebene zum Jura ansteigende Strömung verstärkt bereits über Basel die Bewölkung, so daß im Sommer die Mittellandstationen sonniger sind als die Rheinstadt.

Während sowohl die Winde in Feldberghöhe als auch in der Oberrheinischen Tiefebene und durch die Burgundische Pforte in weniger als 50\% der Fälle dem abgebildeten Trend folgen, dominiert die talauf- und dem Jurakamm zugerichtete Strömung in weit über $50 \%$ der Fälle. Es könnte sein, daß der Sog der besonnten Alpen bis über den Jura hinausgreift und somit den Talaufwind der Juranordseite verstärkt.

Trotzdem ist auch hier eine bedeutungsvolle Alternative vorhanden; nachmittags führt dann eine geschlossene Strömung von Leibstadt über Basel bis Colmar rheinabwärts und erreicht in Muttenz praktisch die gleiche Stärke wie der «normale» rheinaufwärts gerichtete Wind. Auch auf den Paßstationen Staffelegg und Wasserfallen (nicht aber Salhöchi) weht dann der Wind vom Mittelland her über den Jura. Auch für diese Alternativströmung gibt es noch keine eindeutige Erklärung. Eine enge Zusammenarbeit zwischen Einzelbeobachtungen und Modellversuchen J. A. HERTIG (1981) dürfte zur Lösung dieser Frage beitragen können.

Je nach der Strömungsrichtung ergeben sich Gebiete, wo die Windrichtung unbestimmt ist, sei es, daß diese im Windschutz liegen oder daß sich bei Überströmen von Erhebungen im Lee horizontale oder vertikale Wirbel (Rotoren) bilden. Modellflieger nützen den aufsteigenden Ast solcher Gebilde gerne für ihre Flugversuche. Auch bei Strömungsmodellen werden solche Rotoren beobachtet.

\section{Überblick, Strömungsfelder im Sommer (Tabelle)}

Einen kleinen Überblick über die Vielfalt der Strömungsfelder im Raume Basel ergibt die nachfolgende Tabelle für das Sommerhalbjahr. Für die 6 TRW und die Situation mit Nordwind in Colmar als Ergänzung werden getrennt nachts $(04.00$ bis $08.00 \mathrm{Uhr})$ und tags (13.00 bis $17.00 \mathrm{Uhr}$ ) wesentliche Angaben für das häufigste Windfeld gemacht und kursiv für ein weniger häufiges, aber doch mit mehr als $20 \%$ Regelmäßigkeit auftretendes Alternativ-Stromfeld zusammengestellt. Geht die Strömung über einen Jurapa $\beta$ entgegengesetzt als über die beiden andern, so sind beide Richtungen mit / dazwischen angegeben. Es wird noch eine erhebliche Forschungsarbeit benötigen, alle Fälle auf wenige, regelmäßig erhältliche meteorologische Grunddaten zurückzuführen. Für die synoptische Komponente ist es der Luftdruckgradient in 1500 bis $3000 \mathrm{~m}$ Höhe und die vertikale Temperaturverteilung bis $3000 \mathrm{~m}$ Höhe, die als Hauptfaktoren zu werten sind. Für die lokalklimatische Komponente spielen die hier nicht berücksichtigten Strahlungsverhältnisse eine entscheidende Rolle sowie die Aufteilung der Strahlungsbilanz in

a) Erwärmung des Bodens und der Vegetation

b) Verdunstung an den Boden- und Wasseroberflächen und Evapotranspiration durch die Vegetation c) Wärmeabgabe an die Luft.

Nur dieser letzte Anteil c) wirkt sich rasch auf das Strömungsfeld und die Konvektion aus. Die Verdunstungsenergie b) wird erst frei, wenn es zu starker Wolkenbildung und Niederschlag kommt - dann kann die Zirkulation plötzlich verstärkt werden und wird vor allem viel höher hinauf sich auswirken. Je besser es gelingen wird, diese Beziehungen zu ordnen, desto leichter dürfte es auch werden, die Strömungsentwicklung zu prognostizieren.

Schon der Wind auf dem Feldberg weicht oft wesentlich von der Richtung ab, die man nach der Bezeichnung der Wetterlage erwarten würde, insbesondere bei der Nordlage. In der mittleren Höhenschicht muß man unterscheiden zwischen den Paßlagen im Jura (wo nur während 14 Monaten 1979/1980 gemessen wurde) und den Stationen auf Dinkelberg und Plateaujura. Während nachts je nach TRW alle Windrichtungen vorkommen, dominiert tagsüber an fast allen Stationen Nord- bis Nordwestwind. In den Tälern muß man unterscheiden zwischen den Seitentälern (Birs, Ergolz, Frick), dem Hochrheintal von Basel an aufwärts, dem Oberrhein von Basel an abwärts und der Burgundischen Pforte (Belfort). In vielen Fällen ziehen die Stromlinien nicht von Colmar bis Leibstadt durch, sei es nach oben, sei es nach unten. In solchen Fällen werden häufig im Stadtgebiet 


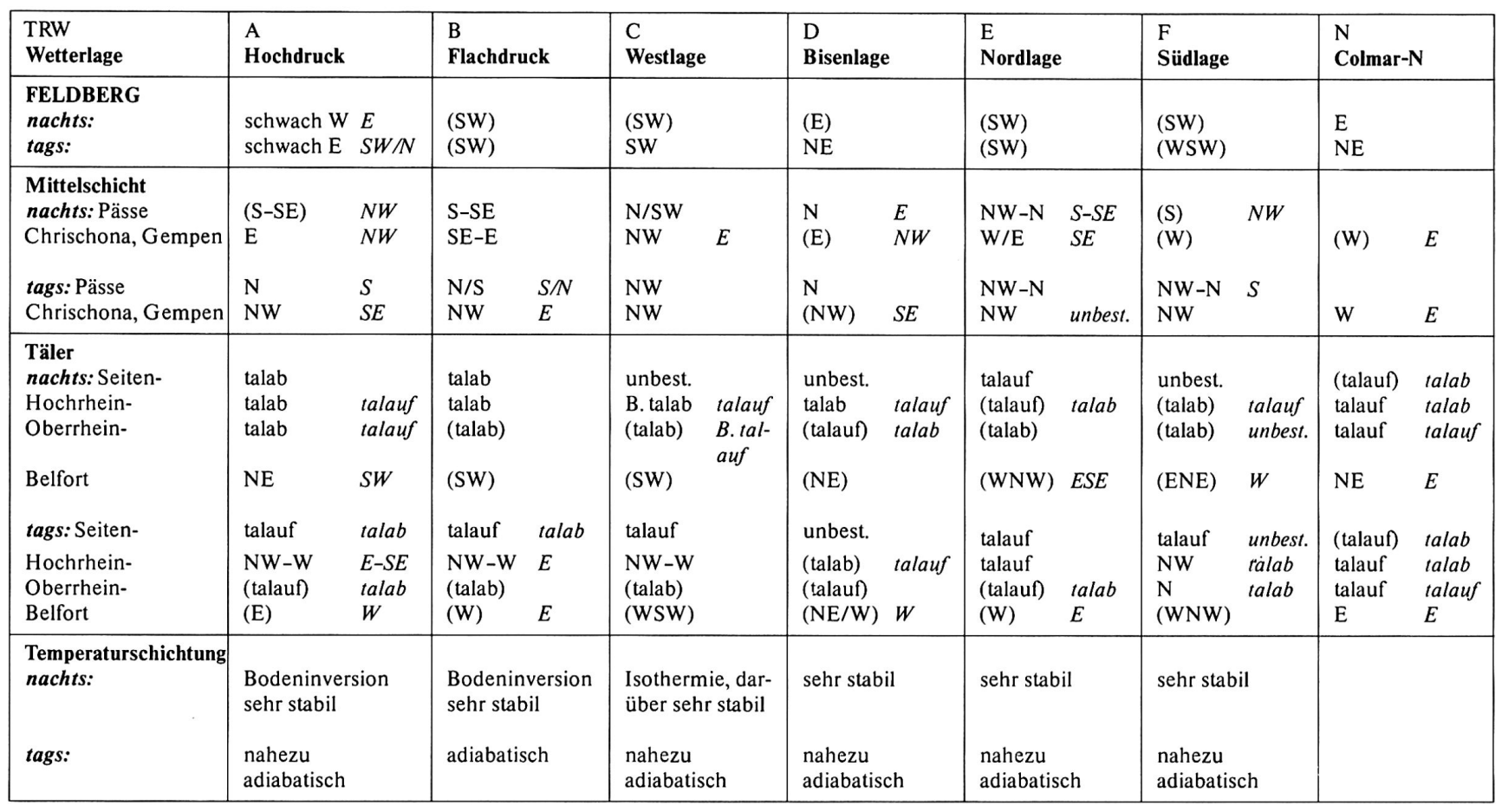


selber verschiedene Windrichtungen beobachtet, was für die Lufterneuerung meist von Nachteil ist. Im speziellen bei der Westlage $\mathrm{C}$ nachts geht die Basler Luft rheinabwärts; während im Hochrhein keine Windrichtung dominiert. Im Alternativfall weht der Wind von Colmar bis Basel inklusive rheinaufwärts, während er gleichzeitig im Hochrheintal bis Muttenz rheinabwärts weht. Als letztes ist für die Burgundische Pforte noch der Wind vom Flughafen von Belfort mitgeteilt, welcher häufig aus jenem Sektor weht, der der Wetterlage entspricht. Zwar wirkt sich noch die Nähe der Vogesen im Norden und in geringerem Maße des Juras im Südosten aus - die Lage ist aber ungleich freier als rund um Basel.

\section{Summary}

During the project CLIMOD (1975-1980) particularly great number of wind-records were collected in the region of Basle. These data arranged in 7 classes of weather and separated for winter and summer, day and night show a great variety of streamfields due to the synoptic wind, the vertical profile of temperature, the flux of solar- and terrestrial radiation. Some phenomena can't be explained by the local scale but will be understood either considering the great volume of cold air in winter between Jura and Alps the "Möhlin-Jet" - or by the strong convergence around the Alps during the long days in summer.

\section{Literatur}

Eidg. Kommission Meteorologie (1981): Möglichkeiten regionaler Klimaveränderungen durch menschliche Einwirkungen. Schlußbericht Projekt CLIMOD, Bern.

BROMBACHER Chr., SCHÜEPP W., WINIGER M. (1979): Der Wetterablauf vom 14. bis 21. Dezember 1977 in der Umgebung Basels. Wiss. Bericht zu Projekt CLIMOD, Basel.

HERTIG J.A. (1981): Modélisation aéraulique de la basse couche atmosphérique, EPF-Lausanne.

HERTIG J.A. (1981): La ventilation régionale dans la vallée du Haut-Rhin, Rhin-Supérieur, résultats de la modélisation physique, EPF-Lausanne.

SCHÜEPP W. (1977): Local winds in medium topography, Proceedings Joint Sc. Meeting AMS, SGBB, SSG Interlaken 1976, Basel.

SCHÜEPP W. (1980): Les champs de brouillard hivernales dans la région de Bâle, Conf. Int. Météorologie Alpine Aix-lesBains 1979, Basel.

WINIGER M. (1982): Klimatische Aspekte des Kernkraftwerkbaus (Studie CLIMOD), In: Geogr. Rundschau 34. 